\title{
Control del fitofago Proarna bergie Distant. por el nematodo Heterorhabditis sp. en el cultivo del espárrago
}

Control of phytophagous Proarna bergie distant. by the nematod Heterorhabditis sp. in the asparagus culture

C. Cedano ${ }^{1}$, A. Julca

\section{RESUMEN}

Objetivo: Evaluar la cepa nativa NE2 de Heterorhabditis sp. sobre las ninfas de Proarna bergie en condiciones de campo, con el fin de reducir la incidencia de este fitófago en las raíces de espárrago que debilitan progresivamente la plantación y reducen el rendimiento. Métodos: Aplicación por inyección al sistema de riego del total de juveniles infectivos de Heterorhabditis sp. desarrollados en larvas de Galleria mellonella utilizando 2 000; 3000 y 4000 larvas por hectárea. Se utilizó un diseño de bloques completos al azar con tres tratamientos más un testigo sin aplicación y tres repeticiones. Se evaluó antes y después de la aplicación el número promedio de ninfas por punto de muestreo por tratamiento. Los datos fueron analizados utilizando el análisis de varianza y la prueba de Tukey. Resultados: todos los tratamientos evaluados afectaron las ninfas de Proarna bergie en campo, destacando la aplicación de 3000 larvas de G. mellonella/ha. con el $57,63 \%$ de mortalidad de las ninfas. Conclusión: La aplicación de juveniles infectivos de Heterorhabditis sp. puede constituirse en alternativa de control biológico sostenible y de bajo impacto en la regulación de ninfas de $P$. bergie Distant. en espárrago.

Palabras clave: Control biológico, nematodos entomopatógenos, juveniles infectivos, mortalidad de ninfas.

\section{ABSTRACT}

Objective: Evaluate the native strain NE2 of Heterorhabditis sp. on the nymphs of Proarna bergie in field conditions, in order to reduce the incidence of this phytophagous in the roots of asparagus that progressively weaken the plantation and reduce the yield. Methods: Application by injection to the irrigation system of the total infectious juveniles of Heterorhabditis sp. developed in larvae of Galleria mellonella using 2,000; 3,000 and 4,000 larvae per hectare. A randomized complete block design with three treatments plus a control without application and three repetitions was used. The average number of nymphs per sampling point per treatment was evaluated before and after application. The data were analyzed using the analysis of variance and the Tukey test. Results: all the treatments evaluated affected the nymphs of Proarna bergie in the field, highlighting the application of 3,000 larvae of G. mellonella / ha. with $57,63 \%$ mortality of nymphs. Conclusion: The application of juvenile infectives of Heterorhabditis $\mathrm{sp}$. It can become an alternative of sustainable and low impact biological control in the regulation of nymphs of $P$. bergie Distant. Asparagus.

Keywords: Biological control, entomopathogenic nematodes, infective juveniles, nymph mortality. 


\section{INTRODUCCIÓN}

La costa peruana presenta un excelente clima y suelo favorables para el cultivo de espárrago lo que permite alcanzar los más altos rendimientos y estándares de calidad a nivel mundial (O'Brien y Díaz, 2004; Gómez y Flores, 2015; Siche y Carranza, 2016 y Cuyuche, Planas, Salazar, 2017). La Libertad concentra aproximadamente el $43 \%$ del área cultivada y produce más de la mitad de la producción nacional (MINAG, 2015; INEI, 2015). Las zonas productoras se ubican principalmente en los valles de Chao, Virú, Moche y Chicama.

En algunos sectores de estos valles los productores refieren la alta incidencia del fitófago Proarna bergi Distant. conocida como cigarra o chicharra, las ninfas de este insecto profundizan en el suelo, se ubican en las raíces, succionan los fotosintatos ocasionando debilitamiento progresivo, menor producción y muerte de plantas (Leal, 2007; Malqui, 2015 y Narro, 2015). El control químico de esta plaga es complicado e ineficiente, requiere elevadas dosis y grandes volúmenes de aplicación del insecticida con el consecuente riesgo de acumulación de residuos tanto en el cultivo como en el suelo.

Los nematodos entomopatógenos (NEPs) son reguladores de plagas que poseen características relevantes como amplio rango de acción, alta virulencia, adaptación y sobrevivencia en condiciones desfavorables y gran capacidad de búsqueda del hospedero por lo que se constituyen en una alternativa de control biológico promisoria en la regulación de plagas, especialmente de aquellas que realizan parte de su ciclo de vida en el suelo (Amador, Molina, Guillen, Parajeles, Jiménez y Uribe; Lizárraga, Castellon y Malqui, 2004; Madrigal, 2001; Rodríguez, HernándezOchandía y Gómez, 2012, Sáenz, 2005, Sáenz y López, 2011; Sáenz, 2011; Sánchez y Rodríguez 2007; Sánchez-Saavedra, Cortez-Madrigal, Cristobal-Acevedo, 2012; Stock ,1996, y Vázquez y Álvarez, 2011).

En áreas esparragueras de Paiján en La Libertad, se han encontrado aislados en nematodos entomopatógenos nativos que por la coloración rojiza de los insectos afectados corresponderían al género Heterorhabditis spp.

Por ello, el objetivo de esta investigación fue evaluar la cepa nativa NE2 de Heterorhabditis sp. sobre las ninfas de Proarna bergie en condiciones de campo.

\section{MATERIALES Y MÉTODOS}

La presente investigación se realizó en la zona denominada La Arenita en el distrito de Paiján en Ascope-La Libertad, en un campo de espárrago del cultivar UC157- F1 de tres años de edad con alto nivel de infestación de ninfas de Proarna bergie Distant.

Los tratamientos consistieron la aplicación de los juveniles infectivos desarrollados en 2 000; 3000 y 4000 larvas de G. mellonella por hectárea, más un tratamiento testigo sin aplicación (Tabla 1).

Tabla 1. Descripción de los tratamientos en estudio

\begin{tabular}{cccc} 
Clave & Descripción Dosis/ha & $\begin{array}{c}\text { Larvas por aplicación y y } \\
\text { frecuencia }\end{array}$ & $\begin{array}{c}\text { Número de } \\
\text { aplicaciones }\end{array}$ \\
\hline T0 & Testigo sin aplicación & - & 2 \\
T1 & $\begin{array}{c}\text { Juveniles infectivos contenidos en } \\
2000 \text { larvas de G. mellonella }\end{array}$ & 1000 /aplicación quincenal & 2 \\
T2 & $\begin{array}{c}\text { Juveniles infectivos contenidos en } \\
\text { 3000 larvas de G. mellonella }\end{array}$ & 1500 /aplicación quincenal \\
T3 & $\begin{array}{c}\text { Juveniles infectivos contenidos en } \\
\text { 4000 larvas de G. mellonella }\end{array}$ & 2000 /aplicación quincenal & 2 \\
\hline
\end{tabular}

Se utilizó un diseño de bloques completos al azar con cuatro tratamientos y tres repeticiones incluidos el tratamiento testigo. La dosis correspondiente a cada tratamiento se aplicó en forma fraccionada en dos oportunidades cada 15 días. Para la aplicación, las larvas de $G$. mellonella que contenían los juveniles infectivos fueron completamente trituradas en forma manual adicionando agua hasta su total desintegración, luego se completó hasta un volumen de 200 litros y se inyectó con bomba estacionaria a través del sistema de riego. Los parámetros evaluados fueron número promedio de ninfas antes de la aplicación y número promedio de ninfas vivas y afectadas después de la aplicación de los tratamientos y porcentaje de mortalidad. 
Los del porcentaje de mortalidad fueron transformados al arcoseno y sometidos a un análisis de varianza y la prueba de medias de Tukey al 0,05 de probabilidad.

\section{RESULTADOS Y DISCUSIÓN}

La densidad de la plaga en el área experimental antes de la aplicación de los JI de Heterorhabditis sp. fue elevada y el promedio por punto de muestreo fluctuó entre 147 a 213 ninfas. A los 45 de la aplicación de los tratamientos se encontró en número variable por tratamiento ninfas muertas y con la coloración rojiza, evidenciando que fueron parasitadas y afectadas por nematodos entomopatógenos del género Heterorhabditis (Figura 1).

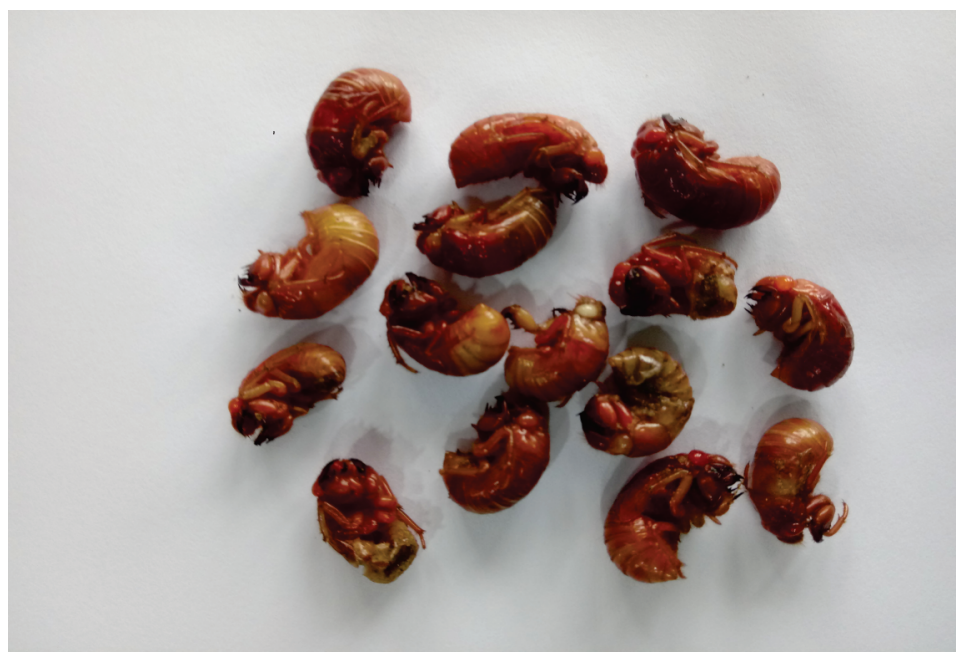

Figura 1. Ninfas de Proarna bergie afectadas por Heterorhabditis sp.

El análisis de variancia de los porcentajes de mortalidad mostró diferencias estadísticas significativas entre los tratamientos, en la prueba de medias de Tukey al $0,05 \%$ se aprecian tres niveles de significancia: Uno conformado por el tratamiento testigo sin aplicación, un segundo grupo por los tratamientos T1 y T3 sin diferencia entre ellos y el tercero con el T2 que alcanzó el mayor porcentaje de mortalidad de ninfas de Proarna bergie y por lo tanto mayor efecto en el control de la plaga.

Tabla 2. Efecto de la aplicación de juveniles infectivos de Heterorhabditis sp. sobre ninfas de Proarna bergie Distant. en espárrago.

\begin{tabular}{ccccc}
\hline Trat. & $\begin{array}{c}\text { Promedio de Ninfas } \\
\text { antes de la aplicación }\end{array}$ & $\begin{array}{c}\text { Promedio de las Ninfas } \\
\text { después de la aplicación }\end{array}$ & $\begin{array}{c}\text { Promedio de } \\
\text { Ninfas afectadas }\end{array}$ & $\begin{array}{c}\text { Mortalidad } \\
\%\end{array}$ \\
\hline T2 & 213 & 105 & 60 & $57,3 \mathbf{~ a ~}$ \\
T3 & 147 & 152 & 68 & $46,7 \mathbf{~ b}$ \\
T1 & 178 & 82 & 20 & $22,3 \mathbf{~ b}$ \\
T3 & 192 & 217 & Sin aplicación & 0,0 c \\
\hline
\end{tabular}

Los porcentajes de control obtenidos son elevados en comparación a los reportados por Narro (2015), que refiere 10,8 y $16 \%$ de control utilizando 60000 y $120000 \mathrm{JI}$ de Heterorhabditis sp. por metro lineal respectivamente, en un campo de espárrago conducido con riego por gravedad. Probablemente estas diferencias se deban a que en esta oportunidad se ha utilizado mayor número de $\mathrm{JI}$ por hectárea y otro factor determinante es la humedad permanente en el campo experimental dado que el cultivo es conducido con riego por goteo.
No existe mayor información sobre el uso de NEPs en el control de ninfas de Proarna bergie u otras especies de cigarras en espárrago, salvo referencias de hallazgos de infección natural como refiere Delgado-Gamboa, Ruíz-Vega, AquinoBolaños, Girón-Pablo (2014) en Sonora - México, en campos cultivados de esparrago en Caborca. Asimismo., Stock, et al. (2009) aislaron de cadáveres de ninfas de la cigarra Dicerorpocta ornea (Homoptera: Cicadidiae) una cepa de Heterorhabditis spp. 
Bajo las condiciones del presente estudio se concluye que la aplicación de juveniles infectivos de Heterorhabditis indica controla eficientemente ninfas Proarna bergie registrando el $57,6 \%$ de mortalidad.

\section{REFERENCIAS BIBLIOGRÁFICAS}

Amador, M., Molina, D., Guillen, C., Parajeles, E., Jiménez, K., \& Uribe, L. (2015). Utilización del nematodo entomopatógeno Heterorhabditis atacamensis cia-ne07 en el control del picudo del banano Cosmopolites sordidus en condiciones in vitro. Agronomía Costarricense, 39, 47-60.

Cuyuche, LA; Planas, JJ; Salazar FA. (2017). Planeamiento Estratégico del Espárrago en el Perú. Tesis para obtener el grado de Magister. Pontificia Universidad Católica del Perú. Lima, Perú.

Delgado-Gamboa, JR; Ruíz-Vega, J; AquinoBolaños, T. \& Girón-Pablo, S. (2014). Revisión de nematodos entomopatógenos aislados en México. Entomología Mexicana 1, 284-288.

Gómez, R. \& Flores, F. (2015). Agricultura y servicios ecosistémicos: El caso del espárrago en Ica. Bol. 42 (77), 09-55. Universidad del Pacífico. Lima, Perú.

Leal, J. (2007). Algunos alcances acerca del comportamiento ninfal de Proarna bergi Distant (Homoptera: Cicadidae) en el cultivo de espárrago. ARENAGRO. Asociación de Agricultores Agroexportadores Propietarios de Terrenos de Chavimochic (APTCH) 3 (7) p. 26.

Lizárraga, T. A., Castellón, M., \& Mallqui, D. (2004). Manejo integrado de plagas en una agricultura sostenible: intercambio de experiencias ente Cuba y Perú. In Taller Internacional (No. H10 T34). Red de Acción en Alternativas al Uso de Agroquímicos, Lima (Peru).

Madrigal, C. A. (2001). Fundamentos de control biológico de plagas. Medellín, Colombia: Universidad Nacional de Colombia, Sede Medellín, Facultad de Ciencias.

Malqui, J. (2015). Efecto de dos concentraciones de Heterorhabditis spp. sobre la mortalidad de ninfas de Proarna bergi Dist. en espárrago UC $157 \mathrm{~F} 2$ en ascope, La Libertad. Tesis para optar el título profesional. Universidad Nacional de Trujillo. Trujillo, Perú.
Ministerio de Agricultura y Riego (MINAG). (2015). Instituto Nacional de Estadística e Informática (INEI). Compendio Estadístico 2015. 88p.

Narro, KA. (2015). Control de Proarna bergi Dist. en espárrago con juveniles de Heterorhabditis spp. en Macabí Alto, Rázuri, La Libertad. Tesis para optar el título de ingeniero agrónomo. Universidad Nacional de Trujillo. Trujillo, Perú.

O'Brien, T. \& Díaz, A. (2004). Mejorando la competitividad y el acceso a los mercados de exportaciones agrícolas por medio del desarrollo y aplicación de normas de inocuidad y calidad: El ejemplo del espárrago Peruano. IICA, San José (Costa Rica) Comisión para la Promoción de Exportaciones, Lima (Perú).

Rodríguez, M. G., Hernández-Ochandía, D., \& Gómez, L. (2012). Nematodos entomopatógenos: elementos del desarrollo histórico y retos para su consolidación como biorreguladores en la agricultura en Cuba. Revista de Protección Vegetal, 27(3), 137-146.

Sáenz, A. (2005). Importancia de los nematodos entomopatógenos para el control biológico de plagas en palma de aceite. Palmas, 26(2), 41-54.

Sáenz, A. (2011). Heterorhabditis sp. SL0708 como una alternativa para el control de plagas [Heterorhabditis sp. SL0708 as an alternative for pest control]. Experiencias en nematodos entomopatógenos: retos y oportunidades de uso en Latinoamérica. Experiences with entomopathogenic nematodes: challenges and opportunities to its use in Latinamerica, 2-12.

Sáenz, A., \& López, J. C. (2011). Ciclo de vida y patogenicidad del aislamiento nativo Heterorhabditis sp. SL0708 (Rhabditida: Heterorhabditidae). Revista Colombiana de Entomología, 37(1), 43-47.

Sánchez, L; Rodríguez, M. (2008). Potencialidades de Heterorhabditis bacteriophora Poinar Cepa HC1 Para el manejo de Hypothenemus hampei Ferr. II. compatibilidad con Beauveria bassiana (Balsamo) Vuillemin y Endosulfan. Rev. Protección Veg. 23(2), 104-111.

Sánchez-Saavedra, MG., Cortez-Madrigal, H; Cristobal-Acevedo, D. (2012). Infectividad de Heterorhabditis indica (Rhabditida: Heterorhabditidae) en adultos y larvas de gallina ciega (Coleoptera: Melolonthidae). 
Revista Chapingo Serie Horticultura 18(3), 383-394.

Siche,R.,\& Carranza, J. (2016). Casos empresariales en agronegocios PerúColombia. Capitulo 3: Caso Espárrago Peruano. RED GCE Red Internacional de Investigación en Gestión del Conocimiento Empresarial. Editorial Lasallista. Consultado el 13 de junio 2019. Disponible en https://www.researchgate.net/profile/Franci sco_Arias_Vargas2/publication/308813_Ca sos_Empresariales_en_Agronegocios_Per u-Cölombia/link/580̄0f4d608ae6c2449f 2 bc a8. pdf\#page $=52$

Sotck, P. (1996). Capacidad patogénica del nematodo entomopatogeno Heterorhabditis argentinensis (Nemata: Heterorhabditidae) frente a insectos asociados con cultivos de alfalfa. Rev. Soc. Entomol. Argent., 55,1-4.
Stock, S. P., Rivera-Orduño, B., \& Flores-Lara, Y. (2009). Heterorhabditis sonorensis $n$. sp.(Nematoda: Heterorhabditidae), a natural pathogen of the seasonal cicada Diceroprocta ornea (Walker)(Homoptera: Cicadidae) in the Sonoran desert. Journal of Invertebrate Pathology, 100(3), 175-184.

Vázquez, L. L., \& Álvarez, J. M. (2011). Control ecológico de poblaciones de plagas. Instituto de investigaciones de sanidad vegetal, Ministerio de la Agricultura. Editorial CIDISAV. La Habana, Cuba.

Correo electrónico:

carolina_cedano@hotmail.com

\section{Revisión de pares:}

Recibido: 20/08/2019

Aceptado: 09/12/2019 